\title{
Fungicidal activity of murine broncho-alveolar macrophages against Blastomyces dermatitidis
}

\author{
A. M. SUGAR, E. BRUMMER* and D. A. STEVENS*
}

Evans Memorial Department of Clinical Research and Department of Medicine, Boston University Medical Center, Boston, MA 02118, and *Division of Infectious Diseases, Departments of Medicine, Santa Clara Valley Medical Center, and Institute for Medical Research, San Jose, CA 95128 and Stanford University, Stanford, CA 94305, USA

\begin{abstract}
Summary. The fungicidal activity of murine broncho-alveolar macrophages (BAM) against the yeast form of a virulent strain of Blastomyces dermatitidis was studied in the small-volume wells of a Terasaki plate. In a 4-h fungicidal assay, significant killing (16$33 \%$ ) of the fungus by unstimulated BAM was demonstrated with BAM from normal mice and from mice rendered immune to lethal infection with $B$. dermatitidis. No significant differences between the activity of BAM from these two sources could be identified. Addition of $10 \%$ autologous normal or immune serum did not augment the macrophage fungicidal activity. Simultaneous experiments with peritoneal macrophages (PM) also gave reproducible killing of the yeast form in the wells of the Terasaki plate, but in the larger wells of microtitration plates, PM showed no significant fungicidal activity. On the other hand, BAM had similar fungicidal activity against $B$. dermatitidis in Terasaki and microtitration-plate wells. The modest fungicidal activity of BAM from immune mice against $B$. dermatitidis suggests that the resistance of immune mice to respiratory challenge is likely to be based on some augmentation of this first line of defence.
\end{abstract}

\section{Introduction}

The mechanisms of resistance to Blastomyces dermatitidis are not completely understood although it has been shown that immune $T$ cells can transfer resistance (Brummer et al., 1982). Using the yeast form of a virulent strain of $B$. dermatitidis (Brass et al., 1982), we have established that peritoneal macrophages (PM) from concanavalin A (con A)-stimulated or immune mice can significantly inhibit the growth of the fungus in a 24-h coculture system compared with PM from unstimulated nonimmune mice (Brummer et al., 1980) and that PM activated by intraperitoneal $B C G$ infection or con A can kill the organism when cultured for $4 \mathrm{~h}$ in an assay that measures the reduction of inoculum colony forming units (cfu) (Brummer et al., 1983). However, the relevant cell with respect to pulmonary infection, the broncho-alveolar macrophage (BAM), had not been investigated. Because a relevant murine model of human blastomycosis (Brass et al., 1982) involves a pulmonary challenge and a primary pneumonia, we have evaluated the

Received 12 Nov. 1984; accepted 8 Jan. 1985.

* Requests for offprints should be sent to: Dr D. A. Stevens, Sta Clara Valley Medical Center, 751 S. Bascom Ave., San Jose, CA 95128 , USA. fungicidal activity of BAMs from normal and immune mice in a previously described assay (Sugar et al., 1983).

\section{Materials and methods}

Mice

Male BALB/cByJIMR mice (Institute for Medical Research, San Jose, CA), 6-12 weeks old, were used in this study. Several experiments were done with BALB/ cByJ mice from Jackson Laboratories (Bar Harbor, ME) with identical results. The mice were pathogen free and housed in a filtered-air environment. Serological tests of the IMR mouse colony for Sendai virus antibody and antibody to other murine viral and mycoplasmal pathogens were negative.

Mice become resistant to $B$. dermatitidis challenge 4 weeks after resolution of subcutaneous infection $(40000$ cfu of the yeast form of $B$. dermatitidis administered to two dorsal sites) (Morozumi et al., 1982). Such mice are referred to as immune or immunised.

\section{Fungus}

The yeast form of a virulent strain of Blastomyces dermatitidis (ATCC 26199), which has been extensively evaluated in this laboratory (Brass et al., 1982; Brummer 
et al., 1983) was grown in $3 \mathrm{ml}$ of chemically defined medium as described by Brummer et al. (1980). The organisms were incubated at $35^{\circ} \mathrm{C}$ on a rotary shaker $(250$ rpm) and subcultured no more than 10 times. Between 48 and $72 \mathrm{~h}$ before an experiment, $c .1 \mathrm{ml}$ of this liquid culture was seeded on to a blood-agar plate, which was incubated at $35^{\circ} \mathrm{C}$. The growth $(2-3$ days) was scraped from the plate with a wire loop and washed once in $4 \mathrm{ml}$ of sterile physiological saline. The numbers of cfu were determined by plating six replicate samples of $1 \mathrm{ml}$ of an appropriate dilution on blood-agar plates.

\section{Broncho-alveolar macrophages (BAM)}

These cells were obtained as described previously (Sugar et al., 1983). In brief, $15 \mathrm{ml}$ of physiological buffered saline with $0 \cdot 1 \%$ ethylenediaminetetracetic acid (EDTA) (J. T. Baker Chemical Co., Phillipsburg, NJ) at $35-37^{\circ} \mathrm{C}$ was used to lavage (i.e., irrigate via the trachea) the lungs of mice that had been killed with ether and exsanguinated immediately before macrophage harvesting. The cells were centrifuged ( $225 \mathrm{~g}$ for $10 \mathrm{~min}$ ) and washed once in Eagle's Minimum Essential Medium with Earle's salts but without L-glutamine (MEM) (Gibco, Grand Island, NY). The pellet was resuspended in complete tissue-culture medium (RPMI-1640, Gibco, containing heat-inactivated fetal bovine serum $10 \% \mathrm{v} / \mathrm{v}$, and penicillin $100 \mathrm{U} / \mathrm{ml}$ plus streptomycin $100 \mu \mathrm{g} / \mathrm{ml}$ ) (TCM). After counting with a haemocytometer, they were suspended at a concentration of $10^{7}$ cells $/ \mathrm{ml}$ and 15 $\mu \mathrm{l}(150000$ cells) were dispensed into each well of a 60 -well Terasaki plate (the plate commonly used for cytotoxicity testing in tissue typing, developed by $\mathrm{Dr}$ P. Terasaki; Robbins Scientific, Mountain View, CA). After incubation for $2 \mathrm{~h}$ at $37^{\circ} \mathrm{C}$ in $\mathrm{CO}_{2} 5 \%$ in air, nonadherent cells were removed from the wells by aspiration and one wash with the medium. The monolayers were then challenged with $B$. dermatitidis as described below.

\section{Peritoneal macrophages ( $P M$ )}

Peritoneal cells were harvested by repeated washes of the peritoneal cavity with $10 \mathrm{ml}$ of MEM containing heparin $10 \mathrm{U} / \mathrm{ml}$ (Brummer et al., 1980). These cells were then treated like the BAM and challenged with $B$. dermatitidis in an identical manner. In selected experiments, monolayers were prepared in 96-well microtitration plates (Microtest II, No. 3040, Falcon, Oxnard, CA) as described by Brummer et al. (1980).

\section{Mouse sera}

When they were killed, mice were bled via the brachial artery as described by Sugar et al. (1983). Serum from normal, unmanipulated mice is referred to as normal mouse serum and that from immunised mice as immune mouse serum (Morozumi et al., 1982).

\section{Measurement of reduction of inoculum cfu (killing)}

Replicate wells (four to six) with or without macrophages were challenged with $0.01 \mathrm{ml}$ of TCM containing $15000 \mathrm{cfu} / \mathrm{ml}$ of the yeast form of $B$. dermatitidis. The cfu of the inoculum was determined at $0 \mathrm{~h}$ by plating an appropriate dilution on blood-agar plates. After $4 \mathrm{~h}$ at $37^{\circ} \mathrm{C}$ in $\mathrm{CO}_{2} 5 \%$ in air, each culture was harvested with a $0.02-\mathrm{ml}$ pipette with a plastic tip and a total volume of $0.06 \mathrm{ml}$ of sterile distilled water. Microscopic examination of the wells in either plates or trays at the end of the harvesting procedure revealed there were neither residual cells nor fungi. The contents of each well were diluted in a final volume of $2 \mathrm{ml}$. The number of $\mathrm{cfu} /$ well was determined by plating $1 \mathrm{ml}$ of the diluted contents on a blood-agar plate and counting the number of colonies after incubation for $4-5$ days at $37^{\circ} \mathrm{C}$. The percentage reduction of inoculum cfu (killing) was calculated by the formula: $(1-[\mathrm{cfu}$ experimental/cfu inoculum] $) \times 100$.

\section{Statistical analysis}

Differences between the means were evaluated by factorial analysis of variance and the Scheffe comparison of differences between means (Dixon and Massey, 1969). Student's $t$ test was used to evaluate selected experiments as noted.

\section{Results}

\section{Characterisation of cell population obtained by lung lavage}

There was no statistically significant difference in the numbers of cells harvested from the lungs of immune mice 4-6 weeks after subcutaneous infecton (mean \pm S.D. of six experiments; $\left.[6.6 \pm 1 \cdot 1] \times 10^{5}\right)$ in comparison with those from normal mice $\left(8 \cdot 5 \pm 2 \cdot 1 \times 10^{5} ; 11\right.$ experiments) (Sugar et al., 1983). However, immune mice had a small but statistically significant decrease in the percentage of macrophages in the lung lavage fluid ( $\mathrm{p}<0.01$ )-e.g., $92 \pm 2 \%$ versus $98 \pm 3 \%$ in normals, based on differential counting of 200 cells/experiment. The remaining cells were lymphocytes, $8 \pm 2 \%$ and $2 \pm 3 \%$, respectively.

\section{In-vitro activity of macrophages against B. dermatitidis}

Experiments were performed with BAM and PM in complete tissue culture medium without added autologous serum or with $10 \%$ normal or $10 \%$ immune-mouse serum, as shown in table I. BAM and PM exhibited similar activity in the wells of the Terasaki plate, with $21-33 \%$ decrease in inoculum cfu (killing). Serum had no modifying effect on the activity of these unstimulated cells. 
Table I. Activity of normal resident broncho-alveolar and peritoneal macrophages against $B$. dermatitidis in the wells of a Terasaki plate

\begin{tabular}{ll}
\hline \multicolumn{1}{c}{ Assay mixture } & $\begin{array}{c}\text { Percentage } \\
\text { killing* }\end{array}$ \\
\hline $\begin{array}{l}\text { B. dermatitidis } \text { plus } \\
\text { Medium only }\end{array}$ & $1 \pm 8(7) \dagger$ \\
BAM & $24 \pm 9(4) \ddagger$ \\
BAM $+10 \%$ fresh normal mouse serum (NMS) & $21 \pm 6(4) \ddagger$ \\
BAM $+10 \%$ fresh immune mouse serum (IMS) & $24 \pm 7(4) \S$ \\
PM & $28 \pm 7(3) \S$ \\
PM + NMS & $33 \pm 10(4) \S$ \\
PM + IMS & $28 \pm 9(4) \S$ \\
\hline
\end{tabular}

${ }^{*}$ Mean $\pm \mathrm{SD}$; number in parentheses is the number of experiments.

$\dagger$ A small increase in the number of cfu occurred during coculture in some experiments.

$\ddagger \mathrm{p}<0.05$ compared to medium control; no other differences between groups significant.

$\S p<0.01$ compared to medium control; no other differences between groups significant.

Table II. Activity of immune broncho-alveolar and peritoneal macrophages against $B$. dermatitidis in wells of the Terasaki plate

\begin{tabular}{ll}
\hline \multicolumn{1}{c}{ Assay mixture } & $\begin{array}{c}\text { Percentage } \\
\text { killing* }\end{array}$ \\
\hline $\begin{array}{l}\text { B. dermatitidis plus } \\
\text { Medium alone }\end{array}$ & 0 \\
BAM (I) $\dagger$ & $20 \pm 11(2) \ddagger$ \\
BAM (I)+NMS & $15(1)$ \\
BAM (I)+IMS & $24 \pm 5 \quad(4) \ddagger$ \\
PM (I) $\dagger$ & $16 \pm 11(2) \ddagger$ \\
PM (I)+NMS & $16(1)$ \\
PM (I)+IMS & $23 \pm 4(4) \S$ \\
\hline
\end{tabular}

\footnotetext{
* See footnote to table I.

$\uparrow$ BAM (I) and PM (I) refer to immune BAM and immune PM, respectively (see text).

$\ddagger \mathrm{p}<0.05$ compared to medium control; no other differences between groups significant.

$\S p<0.01$ compared to medium control; no other differences between groups significant.
}

Results obtained with macrophages from immune mice are shown in table II. Immune BAM and PM had similar activity (15-24\% killing). Again, no significant effect of fresh autologous normal mouse serum (complement) or immune mouse serum (antibody and complement) could be demonstrated.

\section{Comparison of microtitration plate and Terasaki plate}

Having demonstrated fungicidal activity against $B$. dermatitidis by normal and immune peritoneal macrophages in Terasaki-plate wells, and since previous work suggested that these cells were not fungicidal against $B$. dermatitidis in a microtitration plate system, experiments were performed in an attempt to clarify the discrepancy. The effect of increasing the concentration of the cells in the microtitration plate wells, to that approximating the situation in Terasaki wells, was studied. One million cells/microtitration-plate well was studied in the usual $0 \cdot 2-\mathrm{ml}$ volume, and in a volume of $0 \cdot 1$ $\mathrm{ml} /$ well. This had no effect on the PM; no fungicidal activity was observed (data not shown).

The surface areas available for monolayer formation were different in the two systems. With a radius of $0.71 \mathrm{~cm}$, a well in the microtitration plate had a surface area of $0.396 \mathrm{~cm}^{2}$. In contrast, the corresponding radius and surface area in a well of the Terasaki plate were $0.14 \mathrm{~cm}$ and $0.0154 \mathrm{~cm}^{2}$. In a typical experiment, $60 \%$ of $10^{6} \mathrm{PM}$ adhered to a well in the microtitration plate, giving a total cell density of approximately $1.5 \times 10^{6}$ cells $/ \mathrm{cm}^{2}$. In the Terasaki plate, the cell density was approximately $8.3 \times 10^{6}$ cells $/ \mathrm{cm}^{2}$. The possible effect of this increased cell density in the Terasaki plate system was evaluated by adding four times as many cells to the microtitration wells as was usual $\left(4 \times 10^{6}\right.$ cells/ well). A simultaneous assay revealed no significant killing by PM in the microtitration plate in these conditions, but $23 \%$ killing by the same resident PM in the Terasaki plate in the usual conditions. Hence, no obvious reasons for the discordance between the two systems could be demonstrated.

Table III. Fungicidal activity of macrophages against $B$. dermatitidis in wells of microtest plates

\begin{tabular}{lccc}
\hline Assay mixture & $\begin{array}{c}\text { cfu } \\
( \pm \mathrm{SD}) / \\
\text { well }\end{array}$ & killing & (p) \\
\hline B. dermatitidis plus & & & \\
Medium & $322 \pm 40^{*}$ & $8 \%$ & NS \\
PM & $309 \pm 80^{*}$ & $12 \%$ & NS \\
BAM & $254 \pm 80^{*}$ & $28 \%$ & $<0.01$ \\
Con A-PM $\dagger$ & $184 \pm 50^{*}$ & $48 \%$ & $<0.001$ \\
Inoculum $(0 \mathrm{~h})$ & $351 \pm 40$ & $\ldots$ & $\ldots$ \\
\hline
\end{tabular}

$\mathrm{NS}=$ not statistically significant by Student's $t$ test $(\mathrm{p}>0.05)$ against $0 \mathrm{~h}$.

* Quadruplicate cultures measured after $4 \mathrm{~h}$.

$\dagger$ Con A-PM $=$ PM from mice given Con A $100 \mu$ g, i.p., $24 \mathrm{~h}$ earlier. 


\section{Comparison of $B A M$ and $P M$ in microtitration wells}

Fungicidal activity of BAM against $B$. dermatitidis in $0.2-\mathrm{ml}$ wells of microtitration plates was investigated. BAM and resident PM from the same mice were tested in parallel. PM from mice given $100 \mu \mathrm{g}$ of con A intraperitoneally $24 \mathrm{~h}$ earlier were used as positive controls for killing. Results from this experiment are given in table III. BAM, but not $\mathrm{PM}$, significantly reduced the inoculum cfu in this system. BAM activity in microtitration-plate wells $(28 \%)$ was equivalent to that found in smaller Terasaki-plate wells $(21 \% \pm 6 \%)$.

\section{Discussion}

We have demonstrated that BAM from normal mice have a modest $(21 \% \pm 6 \%)$ but significant ability to kill the yeast form of $B$. dermatitidis in the small-volume wells of Terasaki plates. The fungicidal activity of BAM against $B$. dermatitidis in this system was similar to that against $C$. albicans $(18.7 \% \pm 3.1 \%)$ (E. Brummer, A. M. Sugar and D. A. Stevens, unpublished observations). Candidacidal activity in these experiments was greater than that previously reported when killing was assessed by Giemsa staining of cytocentrifuge preparations (Sugar et al., 1983).

The fungicidal activity of murine BAM reported here was equivalent to that reported for BAM from rabbits (Lehrer et al., 1980), but greater than that reported from human BAM (Cohen and Cline, 1971). However, despite the limited fungicidal activity of BAM in man (Cohen and Cline, 1971), rats (Welch, 1981) or mice (Jakab, 1976), BAM have been reported to have good bactericidal activity (Myrvic and Acton, 1977).

It was noteworthy to find equivalent fungicidal activity $(20-30 \%)$ in BAM obtained from normal or immune mice considering how resistant immune mice are to intranasal challenge with the yeast form of $B$. dermatitidis (Morozumi et al., 1982). A more intimate and prolonged association between the organism, immune lymphocytes and macrophages might have increased the fungicidal activity and experiments along these lines are warranted because

\section{REFERENCES}

Brass C, Volkmann C M, Klein H P, Halde C J, Archibald R W R, Stevens D A 1982 Pathogen factors and host factors in murine pulmonary blastomycosis. Mycopathologica 78:129-140. there is evidence for lymphokine modulation of BAM activity (Sone et al., 1981; Warfel and Hadden, 1980).

When comparing the fungicidal capability of BAM with that of PM in wells of Terasaki plates, no significant differences were observed. Results from this comparison parallel those reported on candidacidal ability of rabbit BAM (16\%) and PM $(11 \%)$ (Lehrer et al., 1980) and mice BAM and PM (Sugar et al., 1983). The presence of fresh normal mouse serum (a source of complement) or fresh immune mouse serum (a source of antibody and complement) had no modulating effect on the fungicidal activity of either BAM or PM.

In the present study, different results were obtained when PM were co-cultured with $B$. dermatitidis in $0.02-\mathrm{ml}$ Terasaki-plate wells or $0.2-\mathrm{ml}$ microtitration-plate wells. In contrast to the microtitration-plate system where there was no statistically significant killing (Brummer et al., 1983), PM from normal or immune mice in Terasaki wells were able to kill $20-30 \%$ of the challenge inoculum in a 4$h$ fungicidal assay. On the other hand, BAM had similar fungicidal activity against $B$. dermatitidis in both the small wells of the Terasaki plates and the larger microtest-plate well. Possible explanations for the discordance of results with PM and well size were explored. When corrected for the possible effects of differences in cell concentration or surface area in the microtitration-plate well, no change in fungicidal activity was found. The influence of culture conditions on fungicidal activity of PM warrants further investigation.

The data presented indicate that resident BAM in normal or immune mice present a modest but definite first line of defence against yeast-form $B$. dermatitidis, and suggest that the resistance of immune mice to pulmonary challenge also probably involves other parts of the immune system, such as lymphokines and other factors. Not only may BAM be stimulated to enhance killing, but the ingress of polymorphonuclear leukocytes, which can be induced to kill $B$. dermatitidis (Brummer et al., 1984) participates in the defensive process.

This study was supported in part by a contract from the Office of Naval Research, US Navy, No. N00014-83-K-0018.

Brummer E, Morozumi P A, Stevens D A 1980 Macrophages and fungi: In vitro effects of method of macrophage induction, activation by different stimuli, and soluble factors on Blastomyces. Journal of the Reticuloendothelial Society 28:507-518.

Brummer E, Morozumi P A, Vo P T, Stevens D A 1982 
Protection against pulmonary blastomycosis: Adoptive transfer with T lymphocytes, but not serum, from resistant mice. Cellular Immunology 73:349-359.

Brummer E, Sugar A M, Stevens D A 1983 Activation of peritoneal macrophages by concanavalin A or Mycobacterium bovis BCG for fungicidal activity against Blastomyces dermatitidis and effect of specific antibody and complement. Infection and Immunity 39:817-822.

Brummer E, Sugar A M, Stevens D A 1984 Immunological activation of polymorphonuclear neutrophils for fungal killing: studies with murine cells and Blastomyces dermatitidis in vitro. Journal of Leukocyte Biology 36:505-520.

Cohen A B, Cline M J 1971 The human alveolar macrophage: isolation, cultivation in vitro and studies of morphologic and functional characteristics. Journal of Clinical Investigation 50: 1390-1398.

Dixon W J, Massey F J 1969 Introduction to statistical analysis, 3rd edn. McGraw-Hill, New York.

Jakab G J 1976 Factors influencing the immune enhancement on intrapulmonary bactericidal mechanisms. Infection and Immunity 14:389-398.

Lehrer R I, Ferrari L G, Patterson-Delafield J, Sorrell T 1980 Fungicidal activity of rabbit alveolar and peritoneal macrophages against Candida albicans. Infection and Immunity 28:1001-1008.
Morozumi P A, Brummer E, Stevens D A 1982 Protection against pulmonary blastomycosis: correlation with cellular and humoral immunity in mice after subcutaneous nonlethal infection. Infection and Immunity 37:670-678.

Myrvic Q N, Acton J D 1977 Intracellular mechanisms and extracellular influences. In: Brain J D, Proctor D F, Reid L M (eds) Respiratory defense mechanisms part II. Marcel Decker Inc, New York, pp 1023-1051.

Sone S, Bucana C, Hoyer L C, Fidler I J 1981 Kinetics and ultrastructural studies of the induction of rat alveolar macrophage fusion by mediators released from mitogenstimulated lymphocytes. American Journal of Pathology 103:234-246.

Sugar A M, Brummer E, Stevens D A 1983 Murine pulmonary macrophages: Evaluation of lung lavage fluids, miniaturized monolayers, and candidacidal activity. American Review of Respiratory Diseases 127:110-112.

Warfel A H, Hadden J W 1980 Lymphokine-mediated fusion and migration: inhibition of alveolar macrophages. Experimental and Molecular Pathology 33:153-168.

Welch W D 1981 Unique opsonic requirements of rat pulmonary alveolar macrophages. Current Microbiology 6:315-319.

\section{ADDENDUM AT PROOF STAGE}

In this paper we described the fungicidal activity of resident murine broncho-alveolar (BAM) and peritoneal macrophages (PM) in Terasaki (cytotoxicity testing) plates. We noted that resident PM were not microbicidal for Blastomyces dermatitidis in microtitration plates in the conditions described, confirming earlier work (Brummer et al., 1983). In one experiment presented (table III), BAM appeared to be microbicidal for $B$. dermatitidis in microtitration plates. In experiments performed after acceptance of the paper, we included, as a positive control, demonstration of microbicidal activity for $B$. dermatitidis by BAM activated by lymphokines, as previously described (Warfel and Hadden, 1980; Sone et al., 1981); we have been unable to confirm that resident BAM are microbicidal for $B$. dermatitidis in microtitration plates. Further analysis of the data in table III reveals a larger percentage killing in medium controls in the microtitration-plate experiment than that described in Terasaki plates, and if killing by BAM $(28 \%)$ is compared with that in medium control $(8 \%)$ rather than with the colony forming units (cfu) in the initial inoculum, the killing by BAM would be non-significant. In four subsequent experiments, in which killing in medium controls was $2 \cdot 5 \pm 5 \cdot 0 \%$ (mean $\pm \mathrm{SD}$ ), resident BAM failed to statistically significantly kill the same isolate of $B$. dermatitidis whether comparison was made with medium controls or with inoculum cfu $(1.5 \pm 3.0 \%$ killing). A possible explanation for the results in table III is that $B$. dermatitidis may be more susceptible to killing in inhospitable conditions (where percentage killing by medium controls is high). We now conclude that killing of $B$. dermatitidis by both resident PM and BAM occurs in Terasaki plates but not in microtitration plates. The reasons for this difference are unclear and several possible explanations have been rejected. In contrast, activated PM (Brummer et al., 1983) or activated BAM (E. Brummer and D. A. Stevens, unpublished) kill $B$. dermatitidis in microtitration plates.

E. BRUMMER AND D. A. STEVENS 